\title{
Del carro a la base para bolsones. Notas sobre la construcción compartida de una herramienta para mejorar la calidad del trabajo de recuperadores ambientales de la Ciudad Autónoma de Buenos Aires
}

From the cart to the base for big bags. Notes about the collective construction of a tool to improve the quality of the work of Environmental Recuperators of the Autonomous City of Buenos Aires

Do carro até a base para sacolões. Notas sobre a construção coletiva de uma ferramenta para melhorar a qualidade do trabalho dos Recuperadores Ambientais da Cidade Autônoma de Buenos Aires

Pablo J. Schamber CONICET - Universidad Nacional de Quilmes, Universidad Nacional de Lanús, Universidad Nacional Arturo Jaurétche

Argentina pjschamber@hotmail.com

Juan Pablo Tagliafico CONICET - Universidad Nacional de Quilmes, Universidad de Buenos Aires

Recibido: 04/06/19 Aceptado: 11/10/2019

Resumen. Este artículo describe una experiencia de investigación y transferencia académicas aún en curso sobre el sistema de gestión de los residuos de la Ciudad Autónoma de Buenos Aires (CABA) y de otros municipios del área metropolitana 
Revista Universidad en Diálogo • Vol. 10, N. ${ }^{\circ}$ 1, Enero-Junio, 2020 • 89-108

ISSN 2215-2849 • EISSN: 2215-4752

URL: http://www.revistas.una.ac.cr/index.php/dialogo/index CORREO ELECTRÓNICO: universidadendialogo@una.cr

DOI: https://doi.org/10.15359/udre.10-1.6

bonaerense. Durante el trabajo de campo, se detectaron ciertos inconvenientes en el proceso de trabajo del que participan cooperativas de cartoneros y que tiene lugar bajo un nuevo sistema de gestión de residuos en la CABA. El artículo busca dar visibilidad a dichos inconvenientes, pero principalmente describir el proceso a partir del cual, con la participación de distintos actores, se diseñó, elaboró y puso a prueba una herramienta muy simple que, entendemos, soluciona el problema detectado. Se pretende así contribuir al debate y la reflexión sobre el papel de las actividades de investigación y transferencia académicas en el marco del compromiso con los grupos sociales que son objeto/beneficiarios de las mismas.

Palabras clave: recuperadores ambientales, gestión de residuos, carro, universidad, intervención.

\begin{abstract}
This article describes an ongoing research experience about the waste management system of the Autonomous City of Buenos Aires (CABA) and other municipalities in the metropolitan area of Buenos Aires. During the fieldwork, some inconveniences were detected in the work process that involved cartoneros cooperatives. The article tries to give visibility to these disadvantages. Still, it mainly aims to describe the process by which, with the participation of different actors, a very simple tool was designed, developed, and tested. The aim is to contribute to the debate and reflection on the role of academic research and transfer activities within the framework of the commitment to the social groups that are the object/beneficiaries of these activities.
\end{abstract}

Keywords: environmental recuperators, waste managemen, cart, university, intervention.

Resumo. Este artigo descreve uma experiência de pesquisa e transferência acadêmica em curso sobre o sistema de gestão de resíduos da Cidade Autônoma de Buenos Aires (CABA) e outros municípios da área metropolitana de Buenos Aires. Durante o trabalho de campo, foram detectados alguns inconvenientes no processo de trabalho envolvido pelas cooperativas de catadores e que ocorrem sob um novo sistema de gerenciamento de resíduos na CABA. O artigo procura dar visibilidade a essas desvantagens, mas principalmente descrever o processo a partir do qual, com a participação de diferentes atores e uma ferramenta muito simples resolve o problema detectado. Esta ferramenta foi projetada, desenvolvida e colocada em teste. O objetivo é contribuir para o debate e a reflexão sobre o papel da pesquisa acadêmica e das atividades de transferência no âmbito do compromisso com os grupos sociais que são objeto / beneficiário dos mesmos.

Palavras chave: recuperadores ambientais, gestão de resíduos, carro, universidade, intervenção. 
URL: http://www.revistas.una.ac.cr/index.php/dialogo/index

CORREO ELECTRÓNICO: universidadendialogo@una.cr

DOI: https://doi.org/10.15359/udre.10-1.6

\section{Introducción}

Este artículo describe una experiencia de investigación y transferencia académicas aún en curso sobre el sistema de gestión de los residuos de la Ciudad Autónoma de Buenos Aires (CABA) y de otros municipios del área metropolitana bonaerense, ${ }^{1}$ en la que, sin haber sido parte de los propósitos buscados originalmente, se identificaron inconvenientes relativos a nuevas modalidades de trabajo en la recolección de residuos secos. En consecuencia, se diseñó, elaboró y puso a prueba una herramienta muy simple que entendemos soluciona el problema detectado. Se trata de una estructura metálica circular con cuatro ruedas que puede utilizarse como base para los bolsones de los recuperadores, que no requiere ser transbordada de su ámbito de trabajo y que introduce una serie de mejoras en la práctica de la actividad. Se pretende con las notas sobre este caso contribuir al debate y a la reflexión sobre el papel de las actividades de investigación y transferencia académicas en el marco del compromiso con los grupos sociales que son objeto/beneficiarios de las mismas.

Con la formación del capitalismo industrial en el siglo XIX, el trabajo pasó a ocupar "un lugar de privilegio en la reflexión" general sobre dicho sistema (Hopenhayn, 2002). La separación entre productor y medios de producción hizo visible el proceso de trabajo como objeto de estudio (Marx, 2008; Rieznik, 2001). Luego de la Segunda Guerra Mundial, bajo el desarrollo disciplinar de las ciencias sociales, estos estudios fueron reformulados con el surgimiento y la consolidación de la sociología del trabajo (Castillo, 2000). Desde entonces, es un rasgo común en todos los abordajes, que los medios de producción ocupen un lugar importante como objeto de análisis. En efecto, este ha sido también el caso de los trabajos que, en los últimos veinte años, han estudiado la actividad de cartoneros o recuperadores urbanos en la Argentina (Carenzo, 2014; Gorban, 2014). Presente en todas las descripciones sobre la actividad, nominado según alguna variante de la familia léxica de la palabra carro $^{2}$ (carrito o carreta), dicho elemento es el principal medio de trabajo utilizado para desplazamientos y almacenamiento de lo recolectado. Si bien existe una variedad de modelos en función de los cuales se habilita o restringe

Proyecto PDTS-CIN-CONICET N 594 Convocatoria 2014 "Ambiente, industria e inclusión social. Encadenamientos productivos ligados al reciclaje de residuos sólidos urbanos en el conurbano sur", Universidad Nacional de Lanús (UNLa) y Universidad Nacional de Quilmes (UNQ). Se agradece la colaboración del Lic. Daniel López y su equipo de la Dirección de Patrimonio Histórico de la UNLa la elaboración material de la base y al Arq. Marcelo González los croquis que se adjuntan como parte del anexo.

2 A lo largo del trabajo, utilizaremos las bastardillas en ocasiones para dar cuenta de la utilización de un término nativo. 
Revista Universidad en DiÁlogo • Vol. 10, N. ${ }^{\circ}$ 1, Enero-Junio, 2020 • 89-108

ISSN 2215-2849 • EISSN: 2215-4752

URL: http://www.revistas.una.ac.cr/index.php/dialogo/index CORREO ELECTRÓNICO: universidadendialogo@una.cr DOI: https://doi.org/10.15359/udre.10-1.6

el despliegue de distintas estrategias, ${ }^{3}$ en síntesis se trata de una estructura metálica y/o de madera con ruedas que soporta la carga de los materiales y permite su traslado.

El carro es para los recuperadores una herramienta que incide directamente en el esfuerzo físico, determina cuánto y qué tipo de materiales podrán recogerse y qué espacio geográfico y distancia podrá ser recorrida. Dicho instrumento se relaciona con el valor comercial de los materiales y forma parte del cálculo en la decisión sobre si vale la pena o no recogerlo. En este mismo sentido, en su trabajo sobre cartoneros y cartoneras de José León Suárez, Débora Gorbán (2014) sostiene que el carro se conjuga con el cuerpo, conformando dos elementos fundamentales al momento de decidir sobre cargar o no determinado material.

Ahora bien, en la última década sucedieron una serie de modificaciones en la gestión de los residuos sólidos $\operatorname{secos}^{4}$ en la CABA y, desde el 2014 en particular, comenzó a implementarse el denominado sistema de campanas, ${ }^{5}$ que implicó para los recuperadores involucrados el abandono del uso de sus tradicionales carros (Rocha, 2016). La transformación implicó una serie de mejoras en las condiciones de trabajo de los recuperadores: menos esfuerzo físico, menos horas de trabajo, menos distancias a recorrer, menos material que cargar y, simultáneamente, ingresos regulares y más altos que los obtenidos exclusivamente con la venta de los materiales recolectados y vacaciones pagas.

Quizás debido a que solo se prestó atención a estas novedosas condiciones favorables, el nuevo medio de trabajo que vino a suplir las funciones de los carros fue soslayado, aunque requería atención específica para no darle continuidad a algunos de sus inconvenientes. En efecto, la observación de campo nos permitió registrar que el reemplazo de carros por bolsones de fibra sintética de un metro por lado en la base y más de un metro y medio de alto (big bags) no evita el esfuerzo físico que involucra arrastrarlos, $y$, además, debido a la fricción contra el asfalto a la que es sometido, su vida útil se reduce notablemente. De este modo, desde la incorporación del sistema de campanas pareciera

Por ejemplo, el tipo de carro define la capacidad de carga, pero también la extensión y variedad de recorridos, la posibilidad de trasladar distintos tipos de materiales según su tamaño y peso, o de alternar esta con otras actividades como fletes, comercialización de alimentos, etc. (Saraví, 1994; Schamber, 2008).

4 Los residuos secos, también denominados inorgánicos o reciclables, se diferencian de los húmedos u orgánicos y hacen referencia principalmente a papeles, cartones, plásticos, vidrios, trapos y metales.

5 Se denominan campanas por la forma de pirámide trapezoidal de los nuevos contenedores exclusivos para residuos secos. Dichas campanas son de color verde lima. 
URL: http://www.revistas.una.ac.cr/index.php/dialogo/index

CORREO ELECTRÓNICO: universidadendialogo@una.cr

DOI: https://doi.org/10.15359/udre.10-1.6

que las mejoras en ciertas condiciones de trabajo operaron invisibilizando la problemática que trajo el reemplazo de carros por bolsones de fibra sintética.

En la primera parte de este trabajo se señalan las características centrales de las modificaciones que se dieron en el sistema de gestión de los residuos secos de la CABA y que implicaron el abandono del uso de carros por parte de los recuperadores. Esta descripción general se complementa con un análisis de las nuevas condiciones de trabajo tras las modificaciones en el sistema. Tras identificar algunos inconvenientes y luego de un balance sobre la actividad, se detalla el proceso vinculado al desarrollo de una nueva herramienta cuya elaboración se hizo en la órbita de la Dirección de Patrimonio Histórico de la Universidad Nacional de Lanús (UNLa). Estimamos que dicho desarrollo representó un modo específico de intervenir desde el ámbito universitario en las problemáticas técnicas que presenta el sistema de la actividad (Howard, 1963) que está siendo estudiada. Finalmente, se describen algunas de las proyecciones futuras derivadas de la confección de esta nueva herramienta, sus posibles ventajas y eventuales obstáculos.

\section{De los carros de los RU a los bolsones de los RA: haciendo visible una problemática}

\section{Modificaciones en el sistema de gestión de los residuos secos de la CABA}

Como parte de los acuerdos que habían sido negociados entre las organizaciones de recuperadores y el Gobierno de la CABA(GCABA), luego de que a fines de 2007 la empresa Trenes de Buenos Aires (TBA) en conjunto con el Gobierno nacional clausuraran definitivamente los servicios ferroviarios exclusivos para recuperadores, formaciones conocidas como Tren Blanco o trenes cartoneros (Fraser, 2015); los recuperadores urbanos (RU) mayoritariamente provenientes de municipios del norte del conurbano bonaerense empezaron a disponer de camiones contratados por el GCABA para trasladar sus respectivos carros cargados hasta el lugar de donde eran oriundos.

Los carros eran entregados vacíos en horario vespertino por los recuperadores en cercanías de alguna estación de trenes de los ramales de la línea Mitre en la provincia de Buenos Aires, para que sean subidos a los camiones con los que los trasladaban hacia la CABA. Luego, los recuperadores abordaban los trenes comunes como cualquier pasajero, para encontrarse con sus respectivos carros en determinados sitios de la CABA previamente definidos, que comenzaron a ser identificados como paradas. Allí comenzaban su habitual recorrido recolectando materiales. La tarea de recolección solía demandar no menos de 
Revista Universidad en DiÁlogo • Vol. 10, N. ${ }^{\circ}$ 1, Enero-Junio, 2020 • 89-108

ISSN 2215-2849 • EISSN: 2215-4752

URL: http://www.revistas.una.ac.cr/index.php/dialogo/index CORREO ELECTRÓNICO: universidadendialogo@una.cr

DOI: https://doi.org/10.15359/udre.10-1.6

cuatro horas, al cabo de las cuales el carro cargado era nuevamente entregado en la parada al camión, que lo volvía a trasladar, ahora cargado, hasta el punto de partida en la provincia. Los recolectores por su parte volvían en tren común, se encontraban con sus carros y los llevaban hasta sus respectivas viviendas. Para el traslado de algunos grupos de recuperadores de ciertas paradas, el GCABA contrató además autobuses que hacían el trayecto provincia-CABAprovincia, o al menos alguno de esos tramos.

Al día siguiente, con los carros cargados de la jornada anterior, los recuperadores o algún miembro de su familia descargaba el contenido y procedía a clasificar y acopiar los materiales en determinados espacios de la propia vivienda. El carro descargado estaba listo para comenzar en la tarde una nueva jornada. Las ventas se solían realizar los días sábados, abonando para ello el flete necesario para ir con los materiales al depósito. ${ }^{6}$

Durante el año 2014, a través de "Contratos de Gestión Social para la Prestación del Servicio Público de Recolección de Residuos Sólidos Urbanos Secos" firmados entre la Dirección General de Reciclado (DGREC), dependiente del Ministerio de Ambiente y Espacio Público (MAyEP), y doce cooperativas de recuperadores urbanos. El GCABA les adjudicó zonas exclusivas de la ciudad para la recolección de ese tipo de residuos, infraestructura para su tratamiento (Centros Verdes) y apoyo logístico para el traslado de materiales y personas. Además, se incorporaron al régimen de los incentivos ${ }^{7}$ una cantidad importante de RU. Por su parte, las organizaciones se comprometieron al efectivo cumplimiento de los términos contractuales y a ir incluyendo paulatinamente a recuperadores independientes (no pertenecientes a ninguna cooperativa) que operaran en las zonas adjudicadas.

En el marco de dichos contratos, desde el año 2014 se ha venido desplegando gradualmente en distintos barrios de la CABA una modalidad de recolección selectiva de residuos reciclables que se conoce con el nombre de etapas, y que involucra una serie de elementos en forma simultánea: la instalación del nuevo tipo de contenedor exclusivo para esa clase de residuos (campanas) y la creación de la figura del recuperador ambiental (RA) que pasa a distinguirse del RU principalmente (pero no solamente) porque recibe como incentivo una asignación mensual de casi el doble de pesos.

${ }^{6} \quad$ El trabajo ya mencionado de Débora Gorbán (2014) describe los distintos modos de acopiar y clasificar en distintos espacios del hogar, distinguiendo las situaciones entre aquellos que pueden acopiar y vender semanal o quincenalmente de quienes deben hacerlo diariamente.

7. Incentivos se denomina a un tipo de ingreso que los recuperadores urbanos reciben del GCABA en concepto de retribución por servicios y complemento a los ingresos que perciben por la venta de los materiales que recolectan. 
URL: http://www.revistas.una.ac.cr/index.php/dialogo/index

CORREO ELECTRÓNICO: universidadendialogo@una.cr

DOI: https://doi.org/10.15359/udre.10-1.6

Como se verá a continuación, el cambio al sistema de etapas acarreó importantes modificaciones en la modalidad de trabajo recién descripta. Siempre en horario vespertino, los recuperadores se acercan a las paradas, ya sea por su propia cuenta (generalmente en trenes) o en los autobuses contratados por el GCABA. Una vez allí, el personal de la DGREC denominado responsable de grupo (RG) les toma asistencia. El cobro del incentivo, que se realiza a mes vencido en cuentas bancarias individuales, se relaciona con el presentismo. Es decir, se cobra la totalidad o una parcialidad del incentivo en función de la cantidad de veces que el recuperador se haya presentado a trabajar en la parada.

Una vez dado el presente, el delegado de la parada, que pertenece a la cooperativa, entrega a cada recuperador bolsones y precintos que habían sido previamente descargados en el lugar provenientes del Centro Verde con el que se encuentran vinculados. ${ }^{8}$ Cada recuperador tiene asignada la tarea de recolectar los materiales que los vecinos depositaron en determinadas campanas. En el trayecto entre una y otra campana también retiran material reciclable provisto por algunos clientes (comercios, edificios). Una vez concluido el recorrido que no demanda más de dos horas, cada recuperador cierra su bolsón ajustando con fuerza los extremos superiores del mismo y espera en determinada esquina correspondiente a su zona el paso del camión de la cooperativa. Los operarios que van en el camión suben los bolsones cargados al mismo, mientras que el delegado registra la entrega. El recuperador vuelve a la parada donde espera la salida del transporte contratado por el GCABA que los llevará de vuelta a su respectivo barrio en la provincia, o bien aborda el tren por su cuenta. El camión con la totalidad de los bolsones cargados y la planilla confeccionada por el delegado se dirige al Centro Verde correspondiente, donde otros integrantes de la cooperativa, luego de registrar el peso de cada bolsón y el recuperador responsable del mismo, continuarán esa misma noche o a la mañana siguiente con la tarea de clasificación y preparación de los materiales para la venta, ya sea mediante su enfardado, embolsado o colocación en tachos (como se denomina a los contenedores metálicos que se emplean en la industria de la construcción para el depósito de escombros).

Se puede apreciar cómo la inauguración del sistema de etapas implicó, entre otras modificaciones, la asignación de un recorrido específico (entre campanas) en una zona determinada (adjudicada por contrato a la cooperativa a la que pertenecen los recuperadores), el abandono del empleo y traslado por parte del recuperador de su respectivo carro, y su desentendimiento de las actividades de clasificación y venta. Ahora es la cooperativa la que participa del tratamiento y de la comerciali-

\footnotetext{
Los Centros Verdes son instalaciones acondicionadas para recibir los bolsones cargados con materiales reciclables. Suelen tener tolvas, cintas transportadoras y prensas para enfardar dichos materiales. Cada una de las doce cooperativas que participan del sistema tiene un Centro Verde de referencia.
} 
zación de los materiales en los Centros Verdes de la CABA. Al pasar a ser RA, el recuperador incrementó el monto de su incentivo, pero se transformó el modo en que percibía ingresos por la comercialización de los materiales recogidos. Ahora la cooperativa le abona quincenalmente en la misma cuenta donde percibe el incentivo, un monto variable que se denomina plus por productividad, que guarda relación con el pesaje de los bolsones entregados individualmente.

En función de los comentarios surgidos en entrevistas mantenidas con varios recuperadores que cambiaron de sistema, se aprecia una alta valoración por la mejora en la calidad de vida ocasionada con estos cambios en la modalidad de trabajo. De todas maneras, también existen comentarios que señalan ciertos inconvenientes o desventajas. Se presenta a continuación un cuadro que refleja pros y contras del cambio.

Tabla 1

Pros y contras del nuevo sistema de campanas y RA

\begin{tabular}{|l|l|}
\hline \multicolumn{2}{|c|}{ Nuevo sistema de campanas y RA } \\
\hline \multicolumn{1}{|c|}{ Pros } & \multicolumn{1}{c|}{ Contras } \\
\hline Reducción de horas de trabajo & $\begin{array}{l}\text { Se perdió la posibilidad de recolectar } \\
\text { objetos voluminosos para sí o para } \\
\text { comercializar (electrodomésticos, } \\
\text { muebles, colchones). }\end{array}$ \\
\hline Menor esfuerzo físico & $\begin{array}{l}\text { Desconocimiento del sistema a } \\
\text { través del cual se cobra el plus por } \\
\text { productividad. }\end{array}$ \\
\hline $\begin{array}{l}\text { Disponibilidad de mayor espacio y comodidad en } \\
\text { las viviendas al no llevar materiales. También hay } \\
\text { mayor orden e higiene. }\end{array}$ & Deterioro de los bolsones \\
\hline $\begin{array}{l}\text { Desentendimiento de las ventas de los } \\
\text { materiales, que involucraban el traslado de los } \\
\text { materiales y las negociaciones con el comprador. }\end{array}$ & $\begin{array}{l}\text { Pérdida de la noción de los } \\
\text { valores de la comercialización y }\end{array}$ \\
\hline
\end{tabular}

Fuente: elaboración propia

Algunos datos surgidos del trabajo de campo realizado en una parada específica permitirán apreciar mejor los rasgos principales de la nueva particularidad en el ejercicio de la tarea. 
URL: http://www.revistas.una.ac.cr/index.php/dialogo/index

CORREO ELECTRÓNICO: universidadendialogo@una.cr

DOI: https://doi.org/10.15359/udre.10-1.6

\section{Una parada específica dentro de la etapa}

La etapa pertenece a la Cooperativa Apóstoles, ${ }^{9}$ y cuenta con más de una parada o lugar de encuentro para el inicio y la finalización de las tareas. Cada parada tiene sus respectivos delegados pertenecientes a la organización y su respectivo RG. La parada donde se realizó el trabajo de campo reúne a cuarenta y tres recuperadores y la conforman dos cooperativas: Posadas, cuyo delegado es Lalo, e Iguazú, cuyo referente es Norma. Los cuarenta y tres recuperadores se dividen en veintitrés varones y veinte mujeres. Todas las paradas de esta etapa se vinculan laboralmente con el Centro Verde Candelaria, ubicado en otro barrio de la CABA.

Los recolectores se acercan por sus propios medios a cada parada entre las diecisiete y las dieciocho horas, de lunes a viernes. Generalmente van hasta allí en el tren del ramal Mitre, dado que son oriundos de José León Suárez, localidad de la zona norte del Partido de San Martín. Algunos ya vienen vestidos con la casaca y el pantalón que los identifica como recuperadores de la CABA, mientras que otros las traen en sus mochilas y se las ponen en ese momento. El delegado supervisa que todos cuenten con su respectivo uniforme antes de emprender el recorrido.

En la parada cada recuperador selecciona uno o dos bolsones, que fueron traídos por algún vehículo desde el Centro Verde Candelaria, y suelen estar en la parada antes de que los recolectores empiecen a llegar. Los primeros en arribar eligen los bolsones que se encuentran en mejor estado, $\mathrm{y}$ aprovechan el tiempo para realizarles zurcidas y arreglos varios. Este espacio de preparación antes de la salida para realizar la tarea propiamente dicha sirve para intercambiar información variada entre los miembros de la organización. Por ejemplo, en cierta oportunidad los recuperadores dieron cuenta al delegado de la existencia de demandas por parte de encargados de edificios u otros generadores para ser atendidos, pero se encuentran más allá de su respectivo recorrido, incluso fuera de la zona correspondiente a la etapa. Además, mencionan la existencia de campanas que no están siendo recogidas y de los vecinos que les demandan que las atiendan. Preguntan al coordinador qué hacer, si van o no van, pero este no brinda en ese momento una respuesta precisa.

Una vez registrada la asistencia por parte del RG y retirada la misma cantidad de precintos que de bolsones, los recuperadores se dirigen a realizar su tarea en función de recorridos y campanas pre asignadas. Algunos trabajan de manera individual, otros de a pares. En el precinto se anota el número que en el listado de la planilla tiene cada recolector, y además su nombre. Generalmente, cada recuperador tiene responsabilidad sobre dos o tres campanas, ubicadas a relativamente poca distancia entre sí (100 m aproximadamente). Varios

$9 \quad$ Los nombres reales han sido modificados con el propósito de garantizar el anonimato. 
Revista Universidad en Diálogo • Vol. 10, N. ${ }^{\circ}$ 1, Enero-Junio, 2020 • 89-108

ISSN 2215-2849 • EISSN: 2215-4752

URL: http://www.revistas.una.ac.cr/index.php/dialogo/index CORREO ELECTRÓNICO: universidadendialogo@una.cr DOI: https://doi.org/10.15359/udre.10-1.6

recolectores usan una varilla de fierro de $60 \mathrm{~cm}$ con la punta doblada como un gancho para hurgar dentro de las campanas. Este instrumento les permite traer las cosas desde el fondo de la campana.

Tanto al inicio como al finalizar la carga del bolsón, los recuperadores despliegan una serie de técnicas. Las primeras cajas grandes que aparecen se emplearán para levantar las paredes del bolsón, desplegadas y perpendiculares al piso. Ello permite que el bolsón se mantenga abierto y estable, práctica que fue especialmente tenida en cuenta en el diseño de la base sobre la que aquí se informa, dado que anula la necesidad de un respaldo para sostener abiertos los bolsones. Recién entonces, una vez que están colocadas las paredes, comienza la carga. Siempre que aparezcan cartones grandes y resistentes, se ubicarán detrás de las paredes iniciales para reforzarlas a modo de columnas. Hacia el final de la tarea, antes de cerrar el bolsón, también se ubicarán algunos cartones grandes y sólidos en la superficie y en el techo. Ello impedirá la fuga de materiales más pequeños cuando lo suban y ubiquen dentro del camión.

Los bolsones tienen cuatro orejas que permiten ser levantados por el montacargas. Los recolectores cortan dos de esas orejas en uno de los extremos, de modo que queda una tira larga. La misma se pasará por la otra oreja que no fue cortada y se tira. Con un pequeño cuchillo se harán dos cortes verticales, de modo que la tira pueda pasar por allí y luego anudarse. Luego se coloca el precinto. Se arrastra el bolsón cerrado hasta la esquina donde se aguarda el paso del camión. La tarea ha finalizado.

Hasta junio de 2017 el plus por productividad era un determinado importe $(1,70$ pesos) que se multiplicaba por la cantidad de kilos aportados mensualmente por cada recuperador. Desde entonces se estableció un valor diferencial para quienes logren alcanzar $600 \mathrm{~kg}$ mensuales. Es decir, el precio del kilo se elevó a 3 pesos para aquellos que logren reunir más de $600 \mathrm{~kg}$ al mes, pero se mantuvo en 1,70 pesos para quienes no lo alcancen.

Cada parada cuenta con dos camiones cuyos choferes no pertenecen a la organización, sino que son propietarios (o empleados) de transportistas que prestan el servicio al GCABA. Alrededor de las diecinueve horas los camiones comienzan a dirigirse a ciertas esquinas ya fijadas, donde los recuperadores los esperan con sus bolsones cargados. Cada camión lleva cinco operarios que son también recuperadores que hicieron previamente su propio recorrido cargando su respectivo bolsón, y luego cumplen la función de subir los bolsones al camión. Cobran una suma fija adicional por ser operarios del camión. Según dicen, los lunes son días de mucho trabajo, porque se recolecta lo que se acumuló durante el fin de semana. 
URL: http://www.revistas.una.ac.cr/index.php/dialogo/index

CORREO ELECTRÓNICO: universidadendialogo@una.cr

DOI: https://doi.org/10.15359/udre.10-1.6

En la cabina de uno de los camiones suele ir el delegado de la cooperativa, quien en una planilla anota la cantidad de bolsones cargados de cada recolector que los operarios suben al camión. Cuando el camión se lleva sus respectivos bolsones, los recolectores se dirigen a la parada, donde un colectivo contratado por el GCABA sale hacia José León Suárez a las 20:30 h. Como la organización no quiere perder el privilegio de contar con un transporte exclusivo para volver a sus hogares, existe un sistema de sanciones para los recuperadores que no vuelven juntos. Una vez que se termina el recorrido, el camión con los bolsones se dirige hacia el Centro Verde Candelaria, del que esta parada forma parte desde mediados del año 2016. Entre diciembre de 2017 y junio de 2018, esta parada trabajó 104 días, entre los que logró recolectar 188,238 toneladas de materiales reciclables. La distribución por meses, días trabajados, cantidad de recuperadores y promedio de días trabajados por los recuperadores se presenta en la siguiente tabla:

Tabla 2

Distribución por meses de días trabajados, recuperadores, promedio de días trabajados y kilos

\begin{tabular}{|l|l|c|c|c|c|}
\hline Año & Meses & $\begin{array}{c}\text { Días } \\
\text { trabajados }\end{array}$ & Recuperadores & $\begin{array}{c}\text { Promedio días } \\
\text { trabajados* }\end{array}$ & Kilos \\
\hline 2017 & diciembre & 12 & 42 & 8 & 25.166 \\
\hline 2018 & enero & 15 & 43 & 8,41 & 27.514 \\
\hline & febrero & 16 & 42 & 11,04 & 23.278 \\
\hline & marzo & 20 & 43 & 13,50 & 33.562 \\
\hline & abril & 16 & 42 & 11 & 28.522 \\
\hline & mayo & 15 & 42 & 10,21 & 27.718 \\
\hline & junio & 10 & 41 & 6,8 & 22.478 \\
\hline & Total & 104 & & & 188.238 \\
\hline
\end{tabular}

*El promedio de días trabajados en cada mes se calculó sumando los días efectivamente trabajados de cada recuperador dividida la cantidad de recuperadores que en dicho mes hubiesen entregaron bolsones.

Fuente: elaboración propia.

Si se considera la distribución por sexo del total del material recolectado, se obtiene que las mujeres aportaron el $46 \%$, mientras los varones el $54 \%$ restante. Sin embargo, las mujeres presentan valores más elevados que los de los varones cuando se analizan los pesos promedio por recuperador entre los cinco que más recolectaron y los cinco que menos recolectaron. Así, el peso promedio de recolección de cada recuperador de esta parada fue en ese período de cuarenta y tres kilos, pero si se considera solo a los cinco recuperadores que 
Revista Universidad en Diálogo • Vol. 10, N. ${ }^{\circ}$ 1, Enero-Junio, 2020 • 89-108

ISSN 2215-2849 • EISSN: 2215-4752

URL: http://www.revistas.una.ac.cr/index.php/dialogo/index CORREO ELECTRÓNICO: universidadendialogo@una.cr DOI: https://doi.org/10.15359/udre.10-1.6

más materiales recolectaron el promedio asciende a $76,5 \mathrm{~kg}$ para el caso de los varones y a 78,5 $\mathrm{kg}$ en el caso de las mujeres. También las mujeres tienen el promedio más elevado si se considera a los cinco recuperadores que menos juntaron: $20,2 \mathrm{~kg}$ para las mujeres y $14,7 \mathrm{~kg}$ para los varones.

Resulta interesante notar que casi el $21 \%$ del total del material recuperado por la parada fue recolectado por dos varones y dos mujeres, y también que es levemente mayor el presentismo en el caso de las mujeres que en el de los varones.

\section{Aprendizajes sobre la actividad y los criterios para el diseño}

La posibilidad de diseñar y poner a prueba un instrumento que ayude simultáneamente a disminuir el esfuerzo físico que implica arrastrar los bolsones cargados, permitiendo de este modo: a) disminuir notablemente el esfuerzo físico, b) aumentar la carga, c) mejorar los ingresos individuales y colectivos, d) reducir el acelerado desgaste de los bolsones, disminuyendo los costos de su permanente reemplazo, surgió durante el trabajo de campo orientado por los objetivos de una investigación que procuraba conocer los cambios ocurridos en el sistema que involucra a las cooperativas de recuperadores de la CABA, pero que simultáneamente aspira a tener impacto con alguna contribución a la solución de los problemas sociales de los sujetos con los que se involucra.

A tono con las ideas expresadas por Daniel Mato (2018), se parte de concebir a las universidades públicas en tanto actores sociales comprometidos con las sociedades en las que se insertan. En este mismo sentido, el hecho de que surgiese durante el trabajo de campo y a partir de la observación y la escucha sobre la cotidianeidad de la tarea forma parte de una actitud que transforma al informante en interlocutor (Cardoso de Oliveira, 2004) y entiende que las potenciales transferencias y actividades de extensión son construidas en forma conjunta.

En resumen, en el marco de la investigación sobre el cambio del sistema, se identificó la existencia de un problema y se precisaron ciertos aspectos condicionantes en la búsqueda de soluciones. Por ejemplo, el artefacto que disminuya el esfuerzo físico y permita la realización de los recorridos y la carga de los residuos debería poder encontrarse y dejarse en la parada, al igual que lo que hoy sucede con los bolsones. Pero con la diferencia de que su traslado a los Centros Verdes es innecesario, evitando todos los inconvenientes de logística que actualmente existen entre las paradas y el Centro Verde. Es decir, debería tratarse de elementos que pudiesen permanecer cercanos a las paradas. Fue relativamente sencillo advertir que los anclajes metálicos para las bicicletas, que el propio GCABA viene colocando en distintos lugares de la ciudad desde el 
URL: http://www.revistas.una.ac.cr/index.php/dialogo/index

CORREO ELECTRÓNICO: universidadendialogo@una.cr

DOI: https://doi.org/10.15359/udre.10-1.6

2011 y publicita en su sitio web, que son más de cinco mil en la actualidad (https://www.buenosaires.gob.ar/ecobici/red-ciclovias/estacionamiento), no solo cumplen con dichos requisitos, sino que sería una propuesta difícil de refutar ante eventuales resistencias del propio Gobierno por el uso del espacio público.

Teniendo en cuenta lo descrito, el principal criterio que se tuvo en cuenta a la hora de diseñar la herramienta fue el minimalismo. Es decir, se buscó concebir un objeto que fuese simple, sin elementos sobrantes, liviano, económico, pequeño, pero que al mismo tiempo fuese resistente, pudiera superar la carga promedio de los bolsones llenos y, no menos importante, que no requiriera mantenimiento.

Se buscó que fuese una herramienta de trabajo que no necesitara ser trasladada para su uso por parte del recuperador, como antes sucedía con los carros, y que tampoco requiriera de un lugar espacioso para ser alojada mientras no se ocupa. Es decir, que fuese un instrumento que el recuperador pudiese encontrar en la parada y dejarlo en ella. Además de haberse considerado que pudiese ser asegurada en espacios semejantes a los que el GCABA diseñó como estacionamiento para bicicletas. También se evaluó la posibilidad de que la base pueda ser alojada por un vecino o comercio con interés en colaborar con el recuperador, como de hecho sucedió en el caso del prototipo que se puso a prueba.

\section{La nueva base para bolsones: cronología de los ensayos}

El primer prototipo que se probó ya estaba construido. Fue un rectángulo de $600 \mathrm{~mm}$ y $500 \mathrm{~mm}$, conformado por barras de ángulos de hierro de alas iguales de $12,7 \mathrm{~mm}$, tenía en las esquinas cuatro ruedas de $40 \mathrm{~mm}$ de diámetro hechas de polipropileno y nylon macizas con perno roscado, y se empleaba para soportar y mover un generador eléctrico.

La prueba de este prototipo no dio buenos resultados. Al ser empleado por los recuperadores, las esquinas del bolsón seguían siendo arrastradas en el asfalto y lo mismo ocurrió con el centro del mismo, a medida que iba ganando peso con la carga de materiales; por otro lado, las ruedas quedaban estancadas en las canaletas del escurrimiento superficial de las aguas presente en las calles. Aprendimos que debíamos ganar altura.

Para el segundo ensayo también empleamos un elemento preexistente: una planchuela de hierro doblada y con dos manijas que servían de mango para tambores de chapa de 200 lts. La forma redonda supuso inicialmente algunas dudas respecto de la posibilidad de que pudiese servir para dar estabilidad a un bolsón de base cuadrada, aunque la inexistencia de puntas alimentaba la posibilidad de que se evi- 
Revista Universidad en Diálogo • Vol. 10, N. ${ }^{\circ}$ 1, Enero-Junio, 2020 • 89-108

ISSN 2215-2849 • EISSN: 2215-4752

URL: http://www.revistas.una.ac.cr/index.php/dialogo/index CORREO ELECTRÓNICO: universidadendialogo@una.cr DOI: https://doi.org/10.15359/udre.10-1.6

taran riesgos de choques y fricciones. De este modo, a dicha base redonda de 590 $\mathrm{mm}$ se le soldaron dos manijas más y cuatro ruedas de chapa y goma de $160 \mathrm{~mm}$ de diámetro. De este modo, la base alcanzaba una altura de $195 \mathrm{~mm}$. La prueba de este nuevo prototipo con los recuperadores permitió comprobar que la altura era la adecuada, pero que, por aprovechar un elemento preexistente y estandarizado, no habíamos reparado en el imprescindible aumento del diámetro de la base. Por eso, al prototipo existente se le agregó una planchuela de hierro doblada de $950 \mathrm{~mm}$ de diámetro que fue soldada a través de otros caños a la base de las ruedas. Puesto a prueba con los recuperadores, este nuevo prototipo permitió comprobar que la forma redonda era adecuada y el diámetro correcto. Sin embargo, al haber quedado las ruedas centralizadas por el uso de la planchuela con diámetro más pequeño, los bolsones cargados no conservaban la estabilidad requerida para la tarea.

Finalmente, en función de la experiencia ganada con los diseños y ensayos anteriores, se fabricó el último prototipo, que resultó adecuado (ver anexo). Las ruedas fueron soldadas debajo de chapas rectangulares de $60 \mathrm{~mm}$ por 40 $\mathrm{mm}$ y por fuera del diámetro de la planchuela, de modo tal que se evite el roce con los extremos del bolsón y permita lograr mayor estabilidad. Además, para dar mayor solidez a la estructura y evitar que el centro del bolsón cargado se apoye en el suelo, se soldaron en forma de cruz dos planchuelas del mismo espesor que la que forma el diámetro de la base.

Respecto de su uso, una recuperadora le comentó a su compañera: “Con este carrito se trabaja mucho mejor. Encima es re cómodo para llevar el bolsón y todo, no es pesado ni nada". Durante una de las pruebas, acompañando a las recuperadoras que lo emplearon, se advirtió sobre la complejidad que representaba el momento en que hay que desalojar el bolsón cargado de esta base. Levantarlo no resulta posible debido al peso y volumen, y al volcarlo y sacarlo de la base esta salía disparada hacia cualquier lado. Fue entonces que descubrimos la alternativa de apoyarlo contra el marco de la vereda y empujar el bolsón sobre esta. La experiencia resultó exitosa, el bolsón dejaba su base sin inconvenientes.

Luego de hacer las presentaciones pertinentes al encargado del edificio, en cuyo garaje las recuperadoras que experimentaron la base decidieron guardar cotidianamente la misma, $y$, viendo que por el momento no resultaban necesarias nuevas correcciones al prototipo, decidimos dejársela para que la siguieran utilizando, no sin antes pedirles que informaran respecto de su uso e hicieran saber nuevas ideas para su mejora. Al cabo de varias semanas de emplearlo regularmente, una de las recuperadoras envía el siguiente mensaje de voz por Whatsapp: "Andamos de diez con el carrito. Nos re salva. Anda re bien. Empezá a hacer otra muestra porque esta no te la devolvemos más ehhh, jajajaja". Expresión que fue interpretada como la evaluación de que el prototipo funciona. 
URL: http://www.revistas.una.ac.cr/index.php/dialogo/index

CORREO ELECTRÓNICO: universidadendialogo@una.cr

DOI: https://doi.org/10.15359/udre.10-1.6

De este modo, el desarrollo de la herramienta de trabajo, la nueva base para los bolsones, implicó un proceso de diseño y ensayos que atravesó diversas instancias: cuatro prototipos con sus cuatro respectivos ensayos por parte de recuperadores ambientales que probaron el nuevo diseño en sus jornadas laborales, realizando sus evaluaciones y brindando sus opiniones al respecto. Las diferentes modificaciones que fueron realizándose, desde el prototipo inicial hasta el diseño final, estuvieron basadas en dichas evaluaciones y observaciones propias en campo, hecho que pone de manifiesto un tipo de vínculo particular entre espacios universitarios y científicos y problemáticas en el ámbito laboral. A contramano de aquellas experiencias que, sin ensayos previos, desarrollan un prototipo final o de aquellas otras que basan sus ensayos exclusivamente en los criterios técnicos de los investigadores, la experiencia que relatamos aquí representa un intento por dar valor a los saberes que poseen los actores a partir de su propia práctica en el territorio. Este trabajo, indudablemente más lento, ha tenido como consecuencia lograr el diseño de un dispositivo adecuado para las necesidades de un grupo social concreto que pudo hacer uso del mismo.

A partir de dicha experiencia, elaboramos una propuesta técnica y económica que plantea la posibilidad de ampliar la escala de prueba. Esta propuesta consta de tres aspectos: por un lado, los costos de los materiales, por otro, la capacitación para su elaboración $\mathrm{y}$, por último, el seguimiento y la evaluación. Nos interesa que los miembros de las propias cooperativas que sean identificados como personal de sus respectivos talleres sean quienes, en el marco de capacitaciones que reciban en la Universidad, elaboren las próximas bases. Siguiendo la misma lógica que el proyecto tuvo hasta aquí, la elaboración de nuevas unidades requeriría un nuevo proceso de ensayos y evaluaciones del dispositivo. Su utilización y mantenimiento en una escala mayor posiblemente traiga nuevas complicaciones. Existen aspectos de orden práctico que deben ser ensayados en el territorio: la posibilidad de montar un carro sobre otro, de encastrarlos para reducir el espacio necesario para su depósito, el diseño necesario del lugar para que los carros sean guardados.

Estos son algunos elementos que se presentan como interrogantes previos al ensayo del dispositivo en una escala todavía mayor. Las propuestas fueron remitidas a la Federación Argentina de Cartoneros, Carreros y Recicladores, al Área de Ambiente de la Asociación de Trabajadores del Estado (ATE) de CABA y a funcionarios del GCABA, actores de los que esperamos su interés.

\section{Conclusiones}

A lo largo de este artículo se describió una experiencia de investigación y transferencia académica que aún se encuentra en curso, la cual se centra en el 
Revista Universidad en Diálogo • Vol. 10, N. ${ }^{\circ}$ 1, Enero-Junio, 2020 • 89-108

ISSN 2215-2849 • EISSN: 2215-4752

URL: http://www.revistas.una.ac.cr/index.php/dialogo/index CORREO ELECTRÓNICO: universidadendialogo@una.cr

DOI: https://doi.org/10.15359/udre.10-1.6

sistema de gestión de los residuos de la CABA y de otros municipios del área metropolitana bonaerense. Si bien no fue parte de los objetivos planteados originalmente en el proyecto, la experiencia que brindó la práctica etnográfica y el contacto con los interlocutores en el campo hicieron visible una serie de inconvenientes que se relacionaban particularmente con algunas de las modificaciones producidas tras la nueva modalidad de trabajo en la recolección de residuos secos en CABA. La primera parte se concentró entonces en describir este sistema de etapas y algunas de las importantes modificaciones que trajo consigo, analizando sus ventajas y desventajas. La segunda parte se centró en el diseño, la elaboración y la puesta a prueba de una herramienta que, consideramos, puede solucionar el problema detectado. La estructura metálica circular con cuatro ruedas que constituye la nueva base para los bolsones no requiere ser transbordada de su ámbito de trabajo e introduce una serie de mejoras en la práctica de la actividad de los recuperadores.

El proceso de diseño, elaboración y puesta a prueba del prototipo final (al que se llegó luego de varios diseños y pruebas) se realizó a contramano de aquellas experiencias que basan sus ensayos exclusivamente en los criterios técnicos que poseen los investigadores. Por el contrario, el proceso descrito en este trabajo constituye un intento por dar valor a los saberes que los actores poseen y desarrollan en sus propias prácticas en el territorio. De este modo, representa un particular modo de vincular los espacios universitarios y científicos con las problemáticas laborales, en particular, y sociales, en general. Consideramos que ese vínculo es el que se corresponde con una concepción de las universidades públicas y las actividades científicas comprometidas con las sociedades en las que se insertan y con los grupos sociales con los que se trabaja.

A su vez, este trabajo representa un modo específico de concebir la práctica etnográfica y de dar valor a los saberes y los discursos de los actores, teniendo en cuenta sus demandas y problemas en el territorio.

La elaboración de la nueva base para los bolsones es el resultado de esa forma de pensar la práctica de investigación y el papel de las universidades. Como consecuencia, se ha logrado construir una propuesta técnica y económica para ampliar la escala de prueba. Más allá de algunos elementos que se señalaron como posibles inconvenientes en el futuro, siguiendo con la perspectiva planteada aquí, solo con el contacto con los interlocutores del campo y con nuevas pruebas en el territorio se podrán detectar los nuevos obstáculos y las limitaciones que la propuesta trae consigo. Por lo pronto, la propuesta ha sido extendida a otros actores importantes en el campo y se espera que esto traiga nuevos interrogantes y desafíos. 


\section{Referencias bibliográficas}

Cardoso de Oliveira, R. (2004). El trabajo del antropólogo: mirar, escuchar, escribir. Avá: Revista de Antropología, 5, 55-68. http://sgpwe.izt.uam. $\mathrm{mx} /$ files/users/uami/ana/Lecturas/ROBERTO-CARDOSO.pdf

Carenzo, S. (2014). Lo que (no) cuentan las máquinas: la experiencia sociotécnica como herramienta económica (y política) en una cooperativa de "cartoneros" del Gran Buenos Aires. Antípoda, (18), 109-135. http://www.redalyc.org/articulo.oa?id=81430522006

Castillo, J. (2000). La sociología del trabajo hoy: la genealogía de un paradigma. Trabajo y Sociedad, 2(3) 1-50. http://www.unse.edu.ar/ trabajoysociedad/S-THOYFINAL.htm

Fraser, B. (2015). Urban Railways in Buenos Aires. Spatial and social alienation in the documentary film 'El tren blanco'. Transfers, 5(2), 5-22. https://www. academia.edu/16357401/_Urban_Railways_in_Buenos_Aires_Spatial_ and_Social_Alienation_in_the_Documentary_Film_El_tren_blanco._2015_

Gorbán, D. (2014). Las tramas del cartón. Trabajo y familia en los sectores populares del Gran Buenos Aires. Buenos Aires: Gorla.

Hopenhayn, M. (2002). Repensar el trabajo. Historia, profusión y perspectivas de un concepto. Buenos Aires: Norma.

Howard, A. (1963). Land, activity sistems, and decision-marking models in Rotuma. Ethnology, 2(4), 407-440.

Mato, D. (2018). Repensar y transformar las universidades desde su articulación y compromiso con las sociedades de las que forman parte. $+E$ : Revista de Extensión Universitaria, 8(9), 38-52. https://bibliotecavirtual.unl. edu.ar/publicaciones/index.php/Extension/article/view/7837

Marx, K. (2008). La llamada acumulación originaria. En K. Marx, El capital: el proceso de producción del capital (pp. 891-954). Buenos Aires: Siglo XXI.

Rieznik, P. (2001). Trabajo, una definición antropológica. Razón y Revolución, (7), 1-21. http://www.razonyrevolucion.org/textos/revryr/prodetrab/ ryr7Rieznik.pdf

Rocha, L. (21 de marzo de 2016). Reciclado: habrá cambios en el sistema de campanas verdes. La Nación. https://www.lanacion.com.ar/buenos-aires/ reciclado-habra-cambios-en-el-sistema-de-campanas-verdes-nid1881708 
Revista Universidad en DiÁlogo • Vol. 10, N. ${ }^{\circ}$ 1, Enero-Junio, 2020 • 89-108

URL: http://www.revistas.una.ac.cr/index.php/dialogo/index CORREO ELECTRÓNICO: universidadendialogo@una.cr DOI: https://doi.org/10.15359/udre.10-1.6

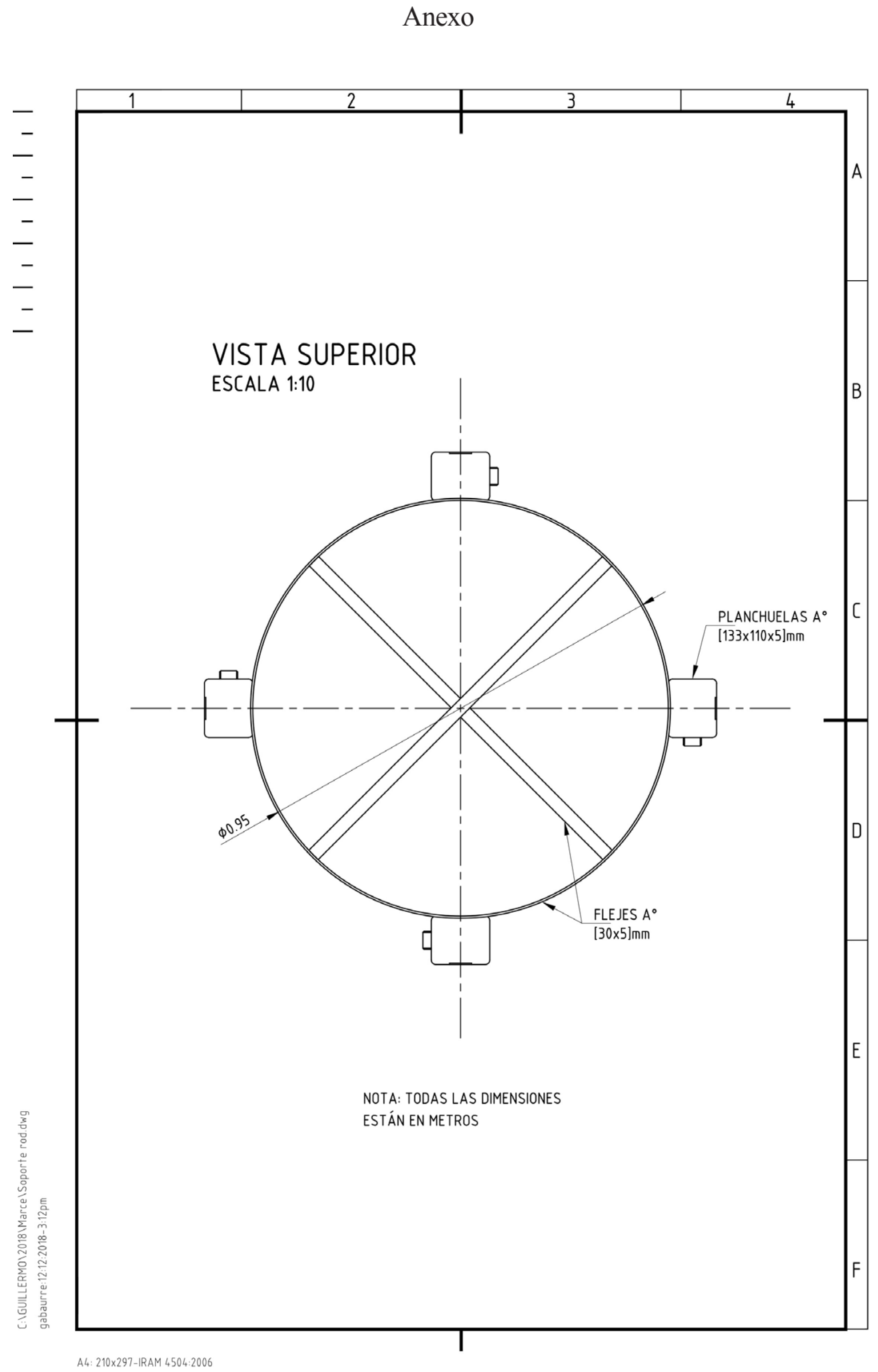


URL: http://www.revistas.una.ac.cr/index.php/dialogo/index

CORREO ELECTRÓNICO: universidadendialogo@una.cr

DOI: https://doi.org/10.15359/udre.10-1.6

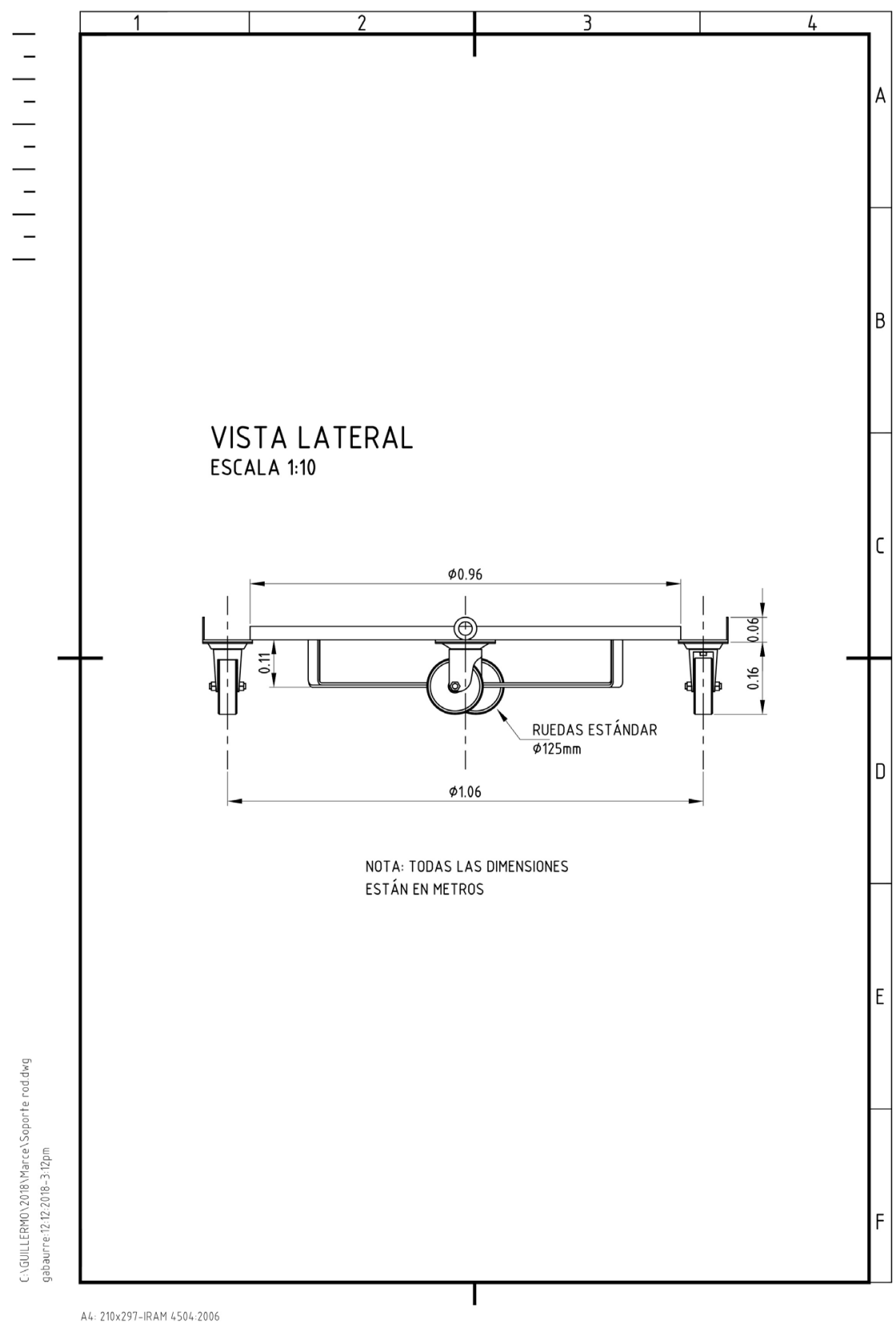


Revista Universidad en Diálogo • Vol. 10, N. ${ }^{\circ}$ 1, Enero-Junio, 2020 • 89-108

URL: http://www.revistas.una.ac.cr/index.php/dialogo/index CORREO ELECTRÓNICO: universidadendialogo@una.cr DOI: https://doi.org/10.15359/udre.10-1.6

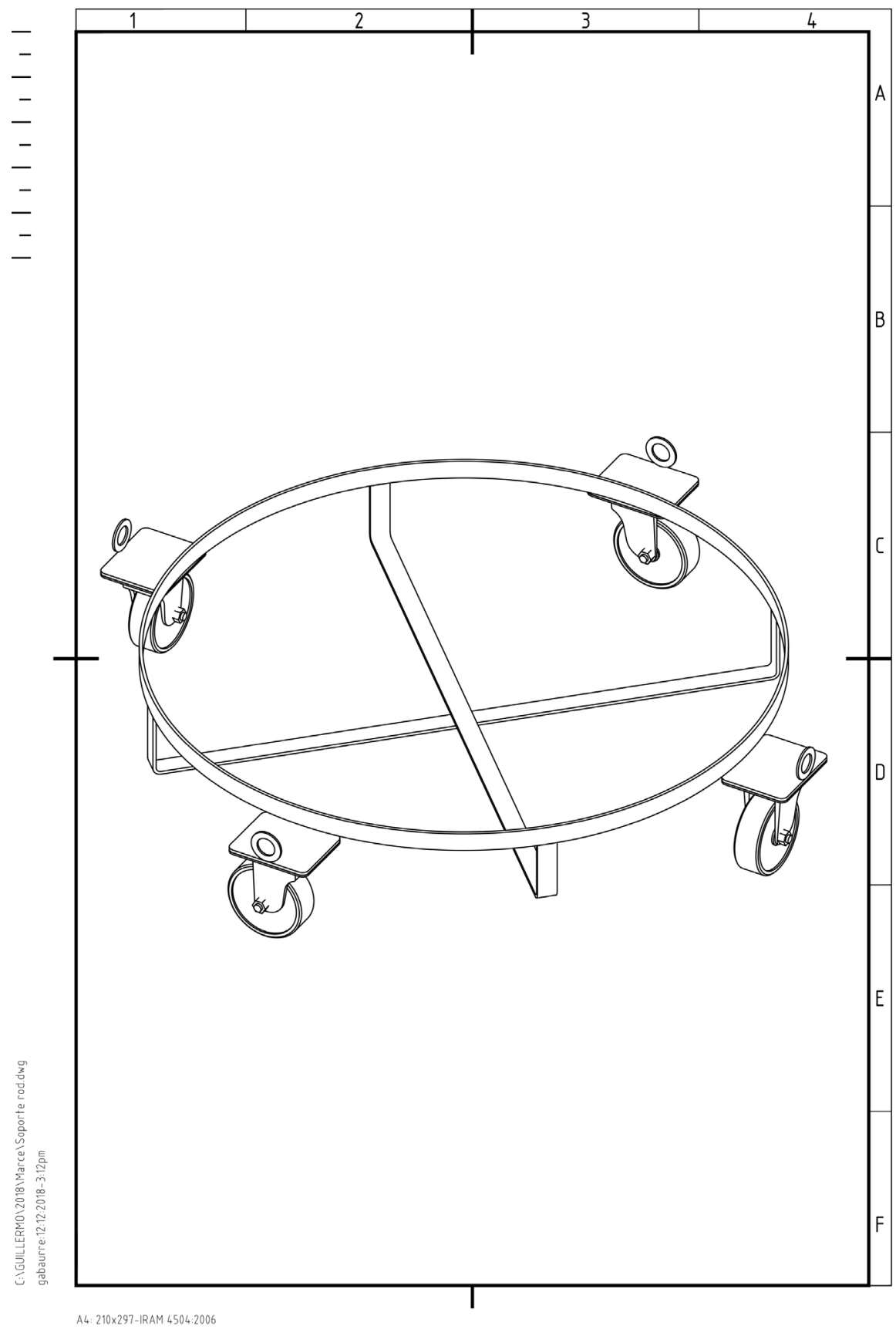

